\title{
High-Resolution Pulmonary Arterio- and Venography Using Multiple-Bolus Multiphase 3D-Gd-MRA
}

\author{
Stefan O. Schoenberg, MD, ${ }^{1,2 *}$ Michael Bock, PhD, ${ }^{2}$ Frank Floemer, MD, ${ }^{2}$ Armin Grau, MD, ${ }^{3}$ \\ David M. Williams, MD, ${ }^{1}$ Gerhard Laub, PhD, ${ }^{4}$ and Michael V. Knopp, MD ${ }^{2}$
}

\begin{abstract}
The purpose of this study was to demonstrate selective visualization of pulmonary arteries and veins with high spatial resolution for improved detection of vascular pathologies. Time-resolved pulmonary three-dimensional gadolinium-enhanced magnetic resonance angiograms (3DGd-MRA) were acquired with an ultrashort 3D fast lowangle shot (FLASH) sequence (TR/TE 2.3/0.9 msec) using asymmetric $\mathbf{k}$-space sampling in readout, phase-encoding, and partition directions. In a single breath-hold four consecutive acquisitions were performed with a scan time of 6.28 seconds for each phase. A spatial resolution of $1.9 \times$ $1.4 \times 2 \mathrm{~mm}^{3}$ could be achieved for a $12 \mathrm{~cm}$ thick 3D volume. To exploit the intrinsic enhancement kinetics of the pulmonary vascular system, the infusion of two gadolinium chelate boli was synchronized with the acquisition of the first and third data sets. This infusion strategy created two predominantly pulmonary arterial and venous data sets with the arterial and venous signal being maximally anti-correlated in time. A further separation of arterial and venous signal could be achieved by means of a correlation analysis. Eight patients with suspected thrombi in the pulmonary veins were studied. The calculated correlation angiographies provided high-quality pulmonary arterioand venograms. Background signal was completely eliminated, allowing maximum intensity projection analysis of the full data set. In $7 / 8$ patients no venous contamination of the arterial phase and vice versa was found. Patency of the pulmonary arteries and veins could be reliably assessed to the level of the segmental vessels. The combination of ultrafast multiphase 3D-Gd-MRA and dual-bolus injection with a correlation analysis of vascular signal provides high-resolution pulmonary arterio- and venograms. This imaging strategy initiates a new competitor to $X$-ray angiography. J. Magn. Reson. Imaging 1999;10:339-346. (c) 1999 Wiley-Liss, Inc.
\end{abstract}

${ }^{1}$ Department of Radiology, University of Michigan, Ann Arbor, Michigan 48109 .

2Department of Radiology, German Cancer Research Center (dkfz), 69120 Heidelberg, Germany.

${ }^{3}$ Department of Neurology, University Hospitals, 69120 Heidelberg, Germany.

${ }^{4}$ Siemens Medical Systems, 91052 Erlangen, Germany.

Contract grant sponsors: Deutsche Forschungsgemeinschaft (DFG), Tumorzentrum Heidelberg/Mannheim, and Verein zur Förderung der Krebserkennung und Krebsvorsorge, e.V.

*Address reprint requests to: S.O.S., Department of Radiology, German Cancer Research Center (dkfz), Im Neuenheimer Feld 280, 69120 Heidelberg, Germany. E-mail: S.Schoenberg@dkfz-heidelberg.de Received July 12, 1999; Accepted July 13, 1999.
Index terms: magnetic resonance angiography; gadolinium; venography; correlation analysis

SELECTIVE VISUALIZATION of the pulmonary arteries and veins in high spatial resolution has been the domain of conventional X-ray angiography. Drawbacks of this technique are its invasiveness, the use of nephrotoxic contrast media, and exposure to ionizing radiation. Magnetic resonance angiography has so far not allowed selective pulmonary angiography. The traditional techniques (including time-of-flight and phasecontrast angiography), with long acquisition times, were substantially limited by motion artifacts, inplane saturation, and intravoxel dephasing. In particular, this affected visualization of small vessel details.

With the introduction of three-dimensional gadolinium-enhanced magnetic resonance angiography (3DGd-MRA), the limitations of non-enhanced MRA were overcome (1). For the first time, high-resolution pulmonary angiograms could be acquired in a single breathhold without use of nephrotoxic contrast media and radiation exposure. 3D-GD-MRA has already been established as a safe and reliable technique for the detection of pulmonary embolism (2). However, overlay of arteries and veins in single-phase acquisitions with scan times of over 20 seconds affects the diagnostic reliability, particularly if assessed by the maximum intensity projection (MIP) algorithm. Several clinical scenarios require a dedicated selective assessment of pulmonary arteries and veins. In 30\% of young patients with cerebrovascular accident (CVA), no underlying etiology is found (3). In these patients, pulmonary venous thrombosis has been suspected as the source of emboli, which was confirmed by autopsy in some cases (4). For accurate surgical planning in patients with pulmonary arteriovenous malformations or bronchial carcinoma, a detailed analysis of the arterial and venous pulmonary vasculature is mandatory.

Multiphase angiography with very short acquisition times in each of the single time-resolved phases has produced pure arterio- and venograms of the lungs at the cost of substantially lower spatial resolution and anatomic coverage (5). In this work, we introduce a new concept for pure arterial and venous pulmonary 3D-GDMRA with high spatial resolution combining multiple 
bolus contrast media injections and multiphase 3D-GDMRA with longer scan times for each single phase.

\section{MATERIALS AND METHODS}

All measurements were performed on a 1.5-T MR system (Magnetom Vision, Siemens Medical Systems, Iselin, NJ), equipped with a resonant echoplanar imaging (EPI) gradient overdrive (maximum gradient strength 25 $\mathrm{mT} / \mathrm{m}$, shortest rise time to maximum $300 \mu \mathrm{sec}$ ). All exams used the standard four-element phased-array body coil, centered on the chest. Aliasing from the patient's arm was suppressed by using copper mesh pillows. The pulmonary arteries were localized with T1-weighted fast low-angle shot (FLASH) breath-hold sequences in coronal and sagittal orientation. Next, multiphase 3D-Gd-MRA was carried out. The anterior margin of the 3D scan volume was positioned at the middle of the long heart axis. Posteriorly, as much of the pulmonary vasculature as possible was covered within the defined scan volume. To obtain pure pulmonary arterio- and venograms, the multiphase 3D-Gd-MRA technique was optimized in terms of acquisition time, contrast infusion, and post-processing as described below.

\section{Multiphase 3D-Gd-MRA Acquisition}

The key issue for time-resolved multiphase pulmonary angiography is the trade-off in spatial resolution and anatomic coverage for shortening of the acquisition time of each phase. Analysis of pulmonary enhancement kinetics shows that in healthy individuals there is only a short time window of approximately 3 seconds during which only the pulmonary arteries are enhanced (Fig. 1). With increasing acquisition time per phase, pulmonary arteries and veins enhance within the same image, and it becomes impossible to separate them on the basis of their enhancement kinetics. The acquisition parameters were therefore set in such a way that in the individual scan phases at least predominant enhancement of either arteries or veins occurred and at the same

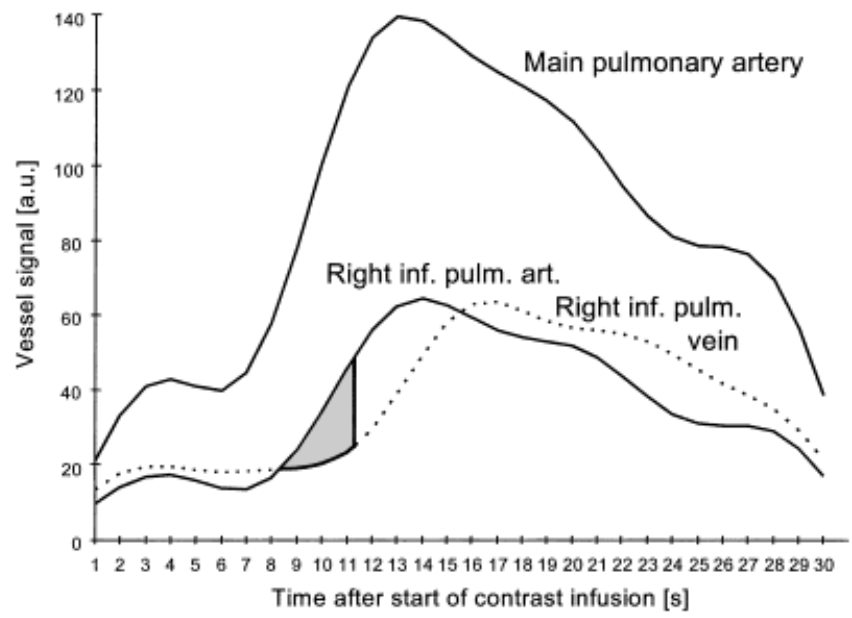

Figure 1. Signal-time course in the pulmonary vascular system after infusion of a test bolus of $1 \mathrm{ml}$ gadopentetate dimeglumine at a rate of $3 \mathrm{ml} / \mathrm{sec}$. There is only a small time window of about 3 seconds (gray area) between substantial enhancement of the right inferior pulmonary artery and the corresponding vein. time spatial resolution and anatomic coverage was maximized. An ultrafast 3D FLASH sequence with asymmetric k-space sampling in readout, phase-encoding, and partition directions and a partially self-refocusing radiofrequency pulse was used (TR/TE $2.3 / 1.1 \mathrm{msec}$, bandwidth $=950 \mathrm{~Hz} /$ pixel). This type of sequence has been previously described in detail (6). In brief, with this sequence only five-eighths of full $\mathrm{k}$-space is acquired, starting about one-eighth before $\mathrm{k}$-space center to preserve some high spatial frequency information. A $26 \times$ $35 \mathrm{~cm}$ field of view and a $12 \mathrm{~cm}$ 3D slab thickness was chosen to be sufficient for anatomic coverage. Within this volume, 90 phase-encoding and 30 partitionencoding steps were performed, resulting in a voxel size of $1.9 \times 1.4 \times 2 \mathrm{~mm}^{3}$ after zero-filling and a scan time of 6.28 seconds. In a single breath-hold four consecutive acquisitions were performed.

\section{Multiple-Bolus Contrast Infusion}

The minimum scan time of 6.28 seconds, which still allows adequate anatomic coverage and spatial resolution, is too long to ensure pure arterial phase angiograms without venous overlay. Therefore separation of arteries and veins was further improved by exploiting the intrinsic enhancement kinetics of the pulmonary system. Previous studies have shown that a smaller dose of contrast media administered with higher infusion rates substantially improves the bolus profile, with faster onset of peak enhancement and faster complete washout at no cost in maximum signal (5). In a multiphasic acquisition, which is perfectly synchronized with the kinetics of the contrast agent, this can be appreciated as a sequential on-off signal course in the pulmonary arteries and veins. If the fast contrast infusion is repeated several times during the odd number of scan phases, a characteristic signal-time course for arterial and venous vessel can be obtained. Arterial signal is at maximum directly after contrast media injection, while venous signal is low. In Fig. 2, arterial signal is decreased and veins appear maximally bright. In the approach presented two contrast boli (each $0.1 \mathrm{mmol} / \mathrm{kg}$ body weight gadopentetate dimeglumine [Magnevist]) were administered by automated infusion (Tomojet CAI, Doltron/Bruker) with an injection rate of $5 \mathrm{ml} / \mathrm{sec}$ (Fig. 2). To ensure high injection rates and a well-defined bolus profile, at least $16 \mathrm{G}$ angiocaths were placed (7). The repetitive injections were synchronized with the first and third scan phase using programmable delays for the start of the pulse sequence and mechanical infusion. The exact delay time (TD) between the start of the infusion and the arrival of the contrast bolus in the main pulmonary artery was determined by a test bolus injection of $1 \mathrm{ml}$ of gadolinium chelate administered during axial single-slice imaging with a TurboFLASH sequence (TR/TE 8.5/4 msec, inversion recovery time $=100$ msec, 1 image acquired per second). With this design, arterial and venous signals are completely anti-correlated in time, enabling complete separation by means of correlation analysis post-processing (Fig. 3).

\section{Cross-Correlation Maps}

The data were transferred to an offline workstation and analyzed with a dedicated post-processing program 


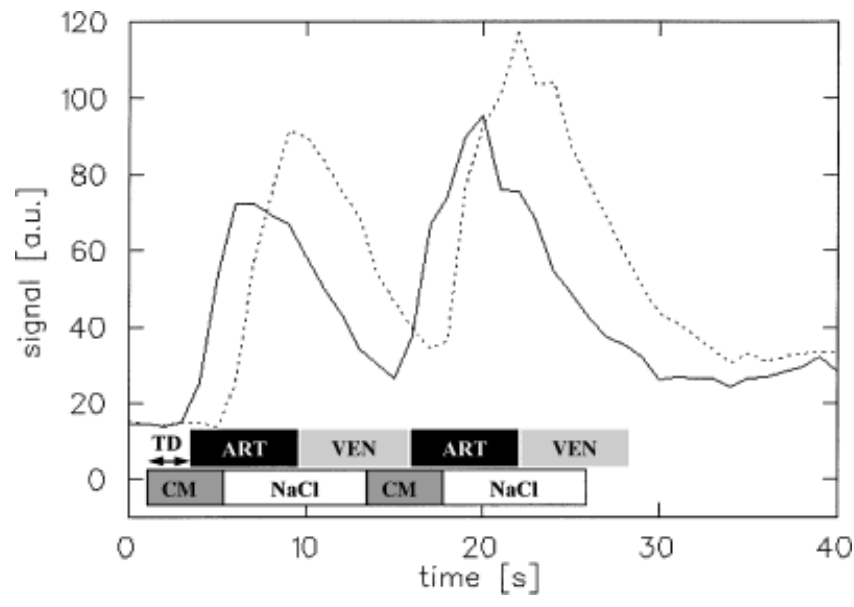

Figure 2. Timing scheme of the multiple-bolus multiphase 3D-Gd-MRA concept. The infusion of two contrast agent boli $(0.1 \mathrm{mmol} / \mathrm{kg}$ bodyweight at $5 \mathrm{ml} / \mathrm{sec})$ is synchronized with the start of the first and third phase (ART) of a multiphasic 3D-Gd-MRA acquisition. A variable time delay (TD) between start of infusion and image acquisition is set to ensure arrival of the contrast media (CM) in the pulmonary arteries at the beginning of the first phase and third phase (ART). This time delay is defined by a prior test bolus sequence using $1 \mathrm{ml}$ of contrast (at $5 \mathrm{ml} / \mathrm{sec}$ ). The signal curve for the test bolus in the main pulmonary artery (continuous line) as well as the ascending aorta (dashed line) is shown. Each contrast infusion is followed by a continuous flush of normal saline (NaCl). During this time the contrast bolus has been propagated into the pulmonary veins, while the second and fourth phase (VEN) of the multiphase scan are acquired.

using a modified correlation analysis (Fig. 4). The correlation algorithm for evaluation of $3 \mathrm{D}$ data sets has recently been described in detail $(8,9)$. In general, correlation analysis allows comparison of a signal-time course in a reference location with that of a target area. If the reference function and the target function for the signaltime course are identical, the correlation is perfect, and the cross correlation coefficient $c$ is 1 . In case of an inverse signal-time course for the reference location and the target location, $c$ equals -1 . In practice, the correlation coefficient $c$ is located somewhere between these two extreme cases of perfect positive or negative correlation. For application of the correlation algorithm to MRA, not the cross correlation coefficient $c$, but the product of $c$ and a scaling factor is used, which reflects the mean signal intensity at this location $(8,9)$. Furthermore, anti-correlated signal-time courses are suppressed by setting all negative signal values to zero. In each location of the four 3D data sets acquired by multiphase 3D-GD-MRA, the modified cross correlation between the local signal-time course and a characteristic arterial or venous reference signal-time course was computed. For the arteriograms, the main pulmonary artery at the level of the bifurcation was mostly chosen as the reference function; for the venograms the right inferior pulmonary vein before entering the left atrium was usually used. An attractive feature of the correlation algorithm, which is maintained in the modified definition, is the intrinsic suppression of static signal. Therefore, background tissue with a constant

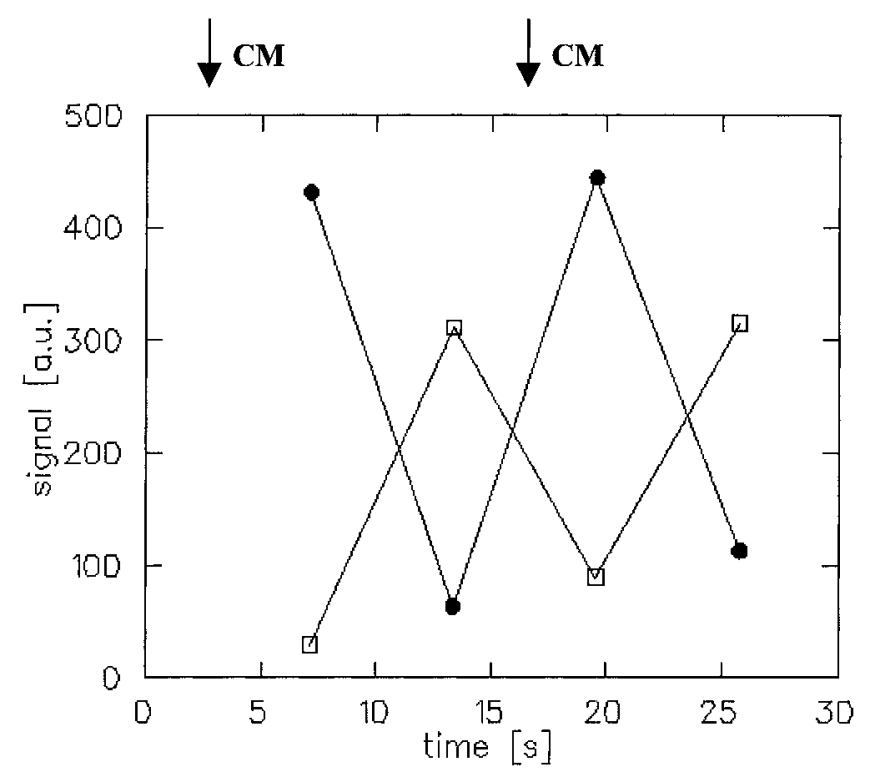

Figure 3. Resulting measured enhancement pattern of pulmonary arteries (๑) and veins $(\square)$ in a series of four multiphasic images using the multiple-bolus concept described in Fig. 2. Note that in the first and third phases of the multiphasic acquisition (at 6 and 19 seconds after sequence start) the arterial signal is at maximum while the venous signal is low. In the second and fourth phases (at 13 and 25 seconds after sequence start) a reversed pattern is found, and arterial and venous signal-time courses are highly anti-correlated. Overall, a slight increase in baseline signal is noted due to recirculation of the contrast agent.

signal-time course appears black in the correlation maps.

\section{Image Analysis}

Coronal and oblique MIP images were calculated from the arterial and venous correlation maps in multiple $15^{\circ}$ angles. For each MIP image the complete 3D data set was used including the marginal partitions, which are

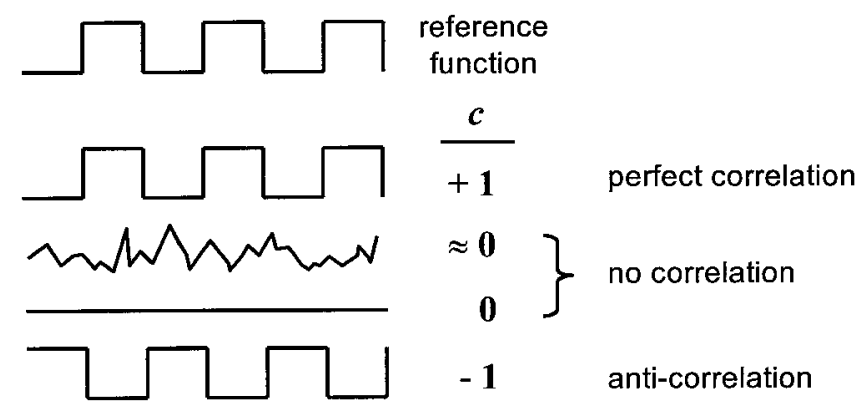

Figure 4. General concept of cross correlation analysis. A signal-time function in a vessel of interest (eg, the pulmonary arteries) is compared with a reference function. This reference function (eg, the main pulmonary artery) is chosen to represent the desired signal-time course, eg, arterial enhancement. If the target function is identical in shape to the reference function a perfect correlation is found and the cross correlation coefficient $c$ is 1 as opposed to an anti-correlation with $c=-1$. In this work, a different scaling factor in the definition of the correlation coefficient is used, which maintains the signal intensities of the MRA data sets in the correlation maps. 
Table 1

Visibility of Pulmonary Arteries in the MIP Images of the Arterial Correlation Maps

\begin{tabular}{cc}
\hline \multicolumn{1}{c}{ Vessel } & Mode score ${ }^{\text {a }}$ \\
\hline Pulmonary arteries & 5 \\
Main & 5 \\
Right and left main & 5 \\
Right and left descending & 5 \\
Right upper lobe & 5 \\
Apical & 5 \\
Posterior & 5 \\
Anterior & 5 \\
Right middle lobe & 5 \\
Medial & 5 \\
Lateral & 5 \\
Right lower lobe & 5 \\
Superior & 3 \\
Medial basal & 5 \\
Anterior basal & 5 \\
Lateral basal & 5 \\
Posterior basal & 5 \\
Left upper lobe & 5 \\
Apical/posterior & 5 \\
Lingual & 5 \\
Lingual superior & 5 \\
Lingual inferior & 5 \\
Left lower lobe & 5 \\
Superior & 3 \\
Medial basal & 5 \\
Posterior basal & 5 \\
Anterolateral basal & 5
\end{tabular}

aVessel visibility defined as grade $1=$ vessel segment not identified, 2 = vaguely identified, 3 = identified, but poorly and incontinuously defined, $4=$ clearly and continuously identified, but indefinite evaluation of patency, and $5=$ clearly identified with definite evaluation of patency.

Table 2

Visibility of Pulmonary Veins in the MIP Images of the Venous Correlation Maps

\begin{tabular}{cc}
\hline Vessel & Mode score $^{\mathrm{a}}$ \\
\hline Right pulmonary veins & 5 \\
Posterior segmental & 5 \\
Apical segmental & 5 \\
Superior pulmonary & 5 \\
Middle lobe & 5 \\
Inferior pulmonary & 5 \\
Superior basal pulmonary & 5 \\
Common basal pulmonary & 5 \\
Left pulmonary veins & 3 \\
Superior pulmonary & 3 \\
Apical segmental & 5 \\
Anterior segmental & 5 \\
Posterior segmental & 5 \\
Middle lobe & 3 \\
Inferior pulmonary & 3 \\
Superior pulmonary & 3 \\
Common basal pulmonary & 3 \\
Basal pulmonary & 3 \\
\hline
\end{tabular}

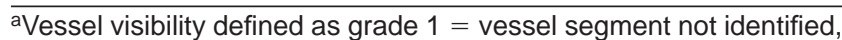
2 = vaguely identified, 3 = identified, but poorly and incontinuously defined, 4 = clearly and continuously identified, but indefinite evaluation of patency, and $5=$ clearly identified with definite evaluation of patency.
Table 3

Overall Vessel Visibility in the Main Vascular Territories of the Lung

\begin{tabular}{cc}
\hline Vessel & Mode score $^{\mathrm{a}}$ \\
\hline Pulmonary arteries & 5 \\
Right upper lobe & 5 \\
Right middle lobe & 5 \\
Right lower lobe & 5 \\
Left upper lobe & 5 \\
Lower lobe & 5 \\
Right pulmonary veins & 5 \\
Left pulmonary veins & 3
\end{tabular}

aVessel visibility defined as grade $1=$ vessel segment not identified, 2 = vaguely identified, 3 = identified, but poorly and incontinuously defined, 4 = clearly and continuously identified, but indefinite evaluation of patency, and $5=$ clearly identified with definite evaluation of patency.

typically omitted in a MIP reconstruction due to incomplete background suppression. The resulting pulmonary arterio- and venograms were assessed by an attending interventional radiologist in terms of vessel visibility, venous/arterial overlay, and breathing/cardiac motion artifacts. Vessel visibility was defined in agreement with previous studies by a semiquantitative score from 1 to 5 (6): $1=$ vessel segment not identified, 2 = vaguely identified, 3 = identified, but poorly and incontinuously defined, 4 = clearly and continuously identified, but indefinite evaluation of patency, and $5=$ clearly identified with definite evaluation of patency. Vessels up to the segmental level were evaluated. Venous/arterial overlay and breathing/cardiac motion artifacts were graded on a scale from 1 to 3 : $1=$ none, $2=$ minor, not affecting a diagnostic evaluation, and $3=$ major, substantially affecting a diagnostic evaluation. The data of all patients were then cumulatively analyzed for each criterion by calculating the mode score.

\section{Study Design}

The study protocol was approved by the institutional review board, and informed consent was obtained for all patients. In an ongoing study, eight patients (five male, three female; age range 31-61 years, mean age $48 \pm 10$ years) have been evaluated. All patients were referred from the Department of Neurology and presented with status post cerebrovascular agents within the previous 7 days. All patients were found negative by extensive diagnostic workup for any potential source of emboli. The workup included Doppler studies of the carotids, a ventilation/perfusion scan, and echocardiography.

\section{RESULTS}

In all patients, the multiple-bolus multiphase approach could be performed, and correlation maps were calculated. The vessel visibility scores for all pulmonary arteries and veins, as assessed by the mode score of all patients, are listed in Tables 1 and 2. In Table 3, the vessels are grouped into major territories. A diagnostic vessel visibility (grade 5) was the most common finding in almost all arterial vessels up to the segmental branches. Only the superior segmental arteries of the right and left lower lobes revealed a substantially lower 

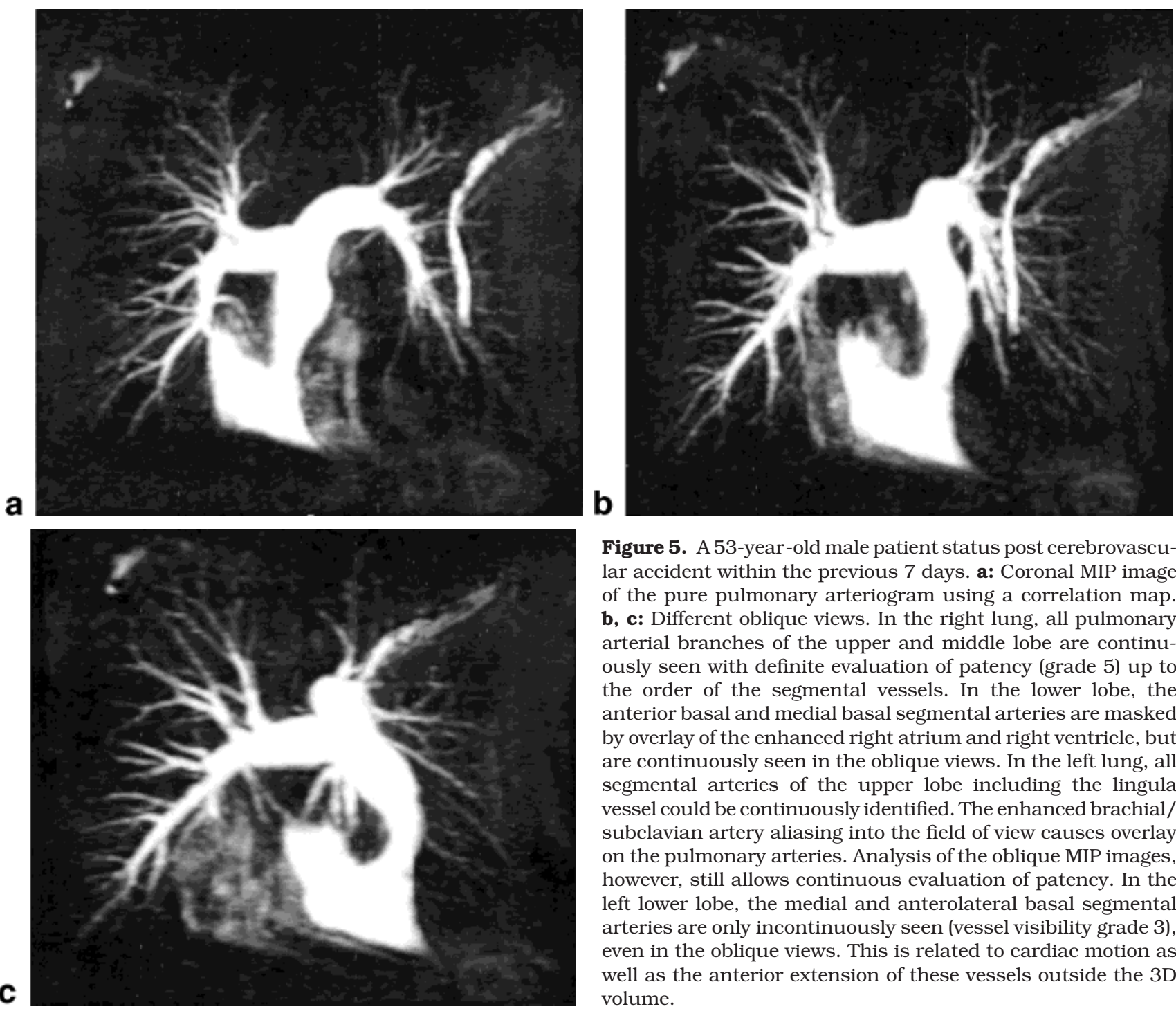

Figure 5. A 53-year-old male patient status post cerebrovascular accident within the previous 7 days. a: Coronal MIP image of the pure pulmonary arteriogram using a correlation map. b, c: Different oblique views. In the right lung, all pulmonary arterial branches of the upper and middle lobe are continuously seen with definite evaluation of patency (grade 5) up to the order of the segmental vessels. In the lower lobe, the anterior basal and medial basal segmental arteries are masked by overlay of the enhanced right atrium and right ventricle, but are continuously seen in the oblique views. In the left lung, all segmental arteries of the upper lobe including the lingula vessel could be continuously identified. The enhanced brachial/ subclavian artery aliasing into the field of view causes overlay on the pulmonary arteries. Analysis of the oblique MIP images, however, still allows continuous evaluation of patency. In the left lower lobe, the medial and anterolateral basal segmental arteries are only incontinuously seen (vessel visibility grade 3), even in the oblique views. This is related to cardiac motion as well as the anterior extension of these vessels outside the 3D volume.

mode score. These vessels were most commonly only identified incontinuously. For the veins, a diagnostic vessel visibility was found for all branches of the right lung. In the left lung, the visibility of pulmonary was overall substantially less, revealing only an incontinuous vessel definition as the most common finding (Tables 2, 3).

Venous overlay on the arterial correlation maps was most commonly considered absent (grade 1) as assessed by the mode score of all patients. In the venograms, no arterial overlay was found. While breathing artifacts were considered only mild, cardiac motion artifacts most commonly substantially degraded (grade 3) the evaluation of the lower lobe vessels. Another artifact limiting the diagnostic evaluation was aliasing of the enhanced brachial/subclavian artery into the field of view cranial to the upper margin of the copper mesh pillow, thereby superimposing on the pulmonary vessels. This was noted in all patients, but it only occurred in the arteriograms since the brachial/ subclavian vein had the same signal-time course as the pulmonary arteries. Similarly, the left ventricle, the aorta, and the greater vessels revealed the same en-

hancement kinetics as the pulmonary veins and thus could not be eliminated in the correlation maps. While the upper pulmonary veins could usually be easily separated from the aorta and greater vessels in oblique views, the lower left veins were commonly indistinguishable from the left ventricle.

In none of the pulmonary veins revealing diagnostic vessel visibility (grade 5) were definite signs of thrombosis, such as hypointensities in the vessel with a fine enhanced rim or interruptions of the vessel with distal reconstitution, found. Complete distal interruptions of vessels close to the margins of the $3 \mathrm{D}$ slab without reconstitution as well as inhomogeneities in vessels with an overall poor visibility score were not taken into account. Figure 5 and 6 show the pulmonary arterioand venogram of a patient in a coronal and an oblique view.

\section{DISCUSSION}

The image quality of 3D-Gd-MRA has remarkably improved within the last few years up to a point at which vascular pathologies are detected with accuracy similar 

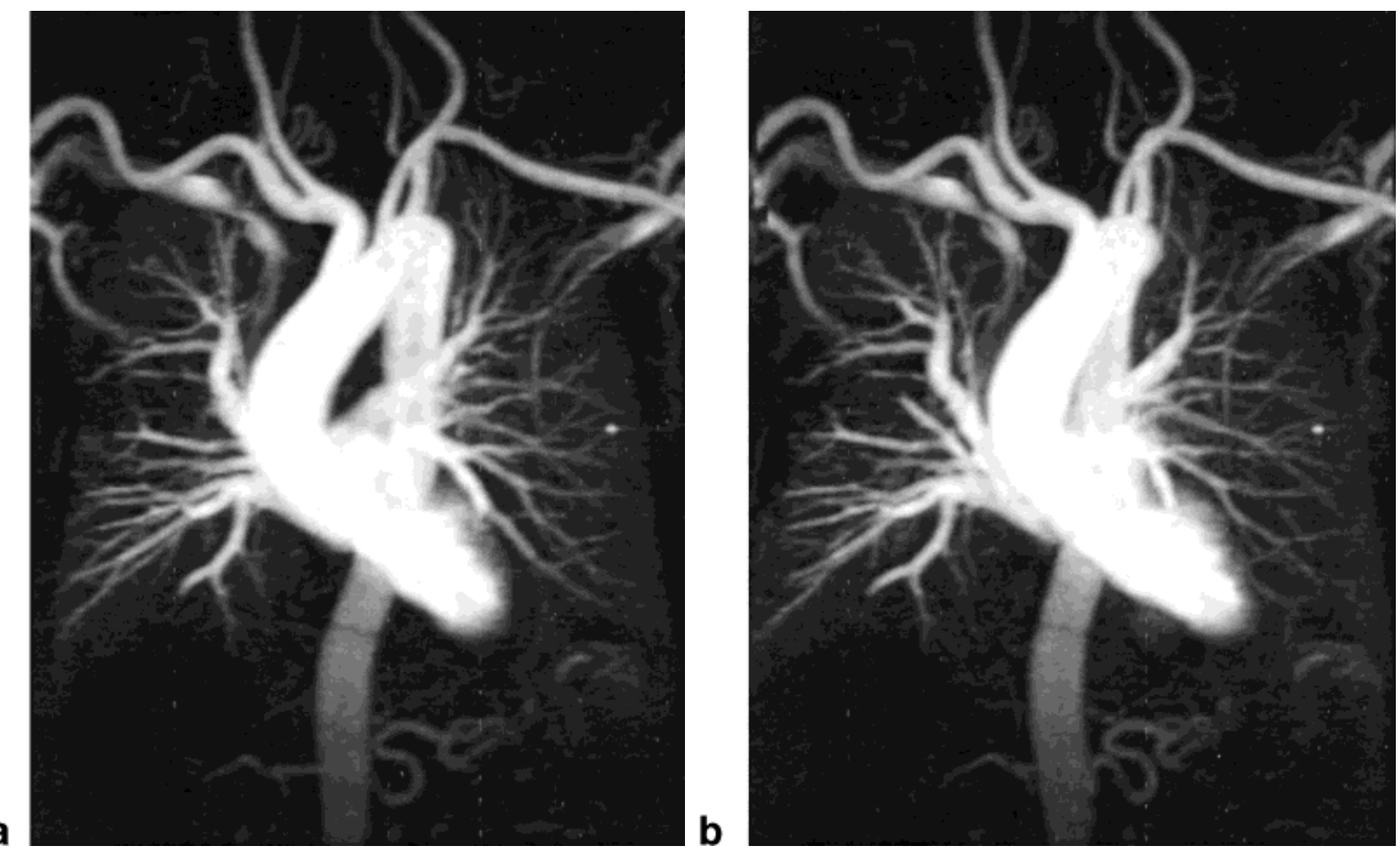

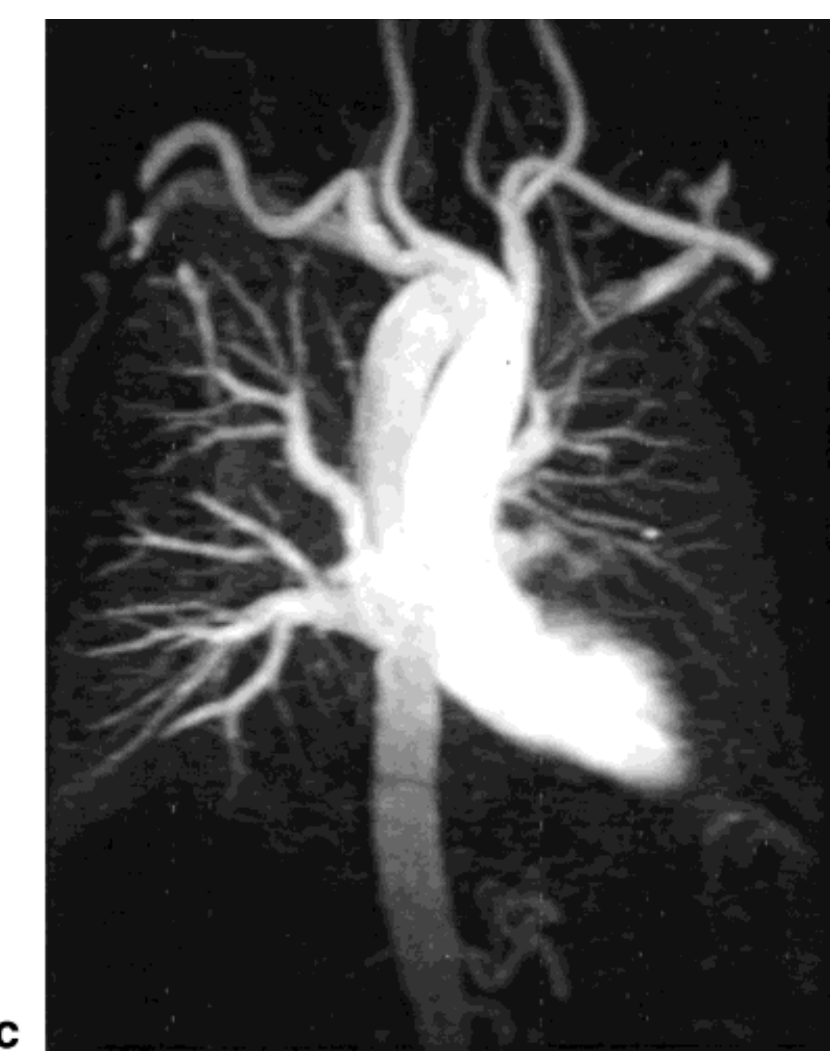

to that in conventional X-ray angiography. This is partially related to the use of faster sequences, which allow higher resolution scans within a breath-hold acquisition. In addition, optimized strategies for bolus timing and acquisition during maximum arterial gadolinium concentrations have substantially contributed to consistently high image quality. However, the prob-
Figure 6. Same patient as in Fig. 5. a: Coronal MIP image of the pure pulmonary venogram using a correlation map. b, c: Different oblique views. Note the excellent visualization of the pulmonary veins without any pulmonary arterial overlay. Only arterial structures with almost identical enhancement kinetics including the left ventricle and the thoracoabdominal aorta with the greater vessels are not eliminated in the correlation maps. In the right lung, all segmental veins of the upper, middle, and lower lobe are continuously seen with definite evaluation of patency. The proximal segments of the right upper, middle, and lower lobe vein are masked by overlay of the ascending aorta in the coronal view but can be definitely evaluated for patency in the oblique views. In the left lungs, all segmental veins including those of the lower lobe reveal a diagnostic vessel visibility. However, overlay of the descending aorta masks the evaluation of the left central veins, even in the oblique views. No signs of thrombi were found in the vessels with diagnostic visibility. The bright spot in the left lung is a centerline artifact, which could not be completely suppressed by the correlation algorithm.

lem remained of imaging separately structures with rapid sequential enhancement. This includes imaging of pulmonary arteries without overlay of veins or renal arteries without overlay of renal parenchyma.

Ultrafast multiphasic acquisition addresses this issue by sequential acquisition of a multiple 3D data set during various stages of contrast enhancement $(5,6)$. 
This time-resolved approach was originally introduced on systems with low gradient performance and therefore long repetition times. Extensive post-processing was necessary to interpolate the few sampled data to several time frames (10). With the availability of highperformance gradients, multiphase angiography has become feasible without long reconstruction times. The use of ultrashort repetition times of less than $4 \mathrm{msec}$ allows direct sampling of the data at a much higher rate. In combination with asymmetric $\mathrm{k}$-space sampling, the acquisition time can be further reduced at only a minor cost to spatial resolution. In previous studies, multiphasic acquisitions have demonstrated encouraging results for the acquisition of pure pulmonary arteriograms.

One drawback of multiphasic acquisitions is that the high frame rate does not allow the same spatial resolution to be obtained as in single-phase studies. This becomes particularly important if the time window between the enhancement of different vessels is extremely short, such as in the pulmonary system. In the concept presented the acquisition time per time frame was therefore extended to twice the time of pure arterial enhancement. To maintain separation between the arterial and mixed arteriovenous phase, the intrinsic enhancement kinetic of the pulmonary vascular system was exploited as a second discriminating criterion. While large volumes of contrast with low infusion rates result in an almost sequential enhancement of pulmonary arteries and veins, smaller doses with high infusion rates create an anti-correlating enhancement pattern of pulmonary arteries and veins. Based on this enhancement pattern, pure pulmonary arterio- and venograms could be extracted by means of a correlation analysis.

Correlation analysis is widely used in neuro-functional MRI (fMRI) to reduce the amount of data acquired in a fMRI time series and to quantify the statistical significance of the results (11). Recently, correlation analysis has been introduced to time-resolved 2D and 3D multiphasic acquisitions after contrast agent injection $(8,9,12)$. Here, correlation analysis is used to compare the signal-time course of a vessel structure of interest with a reference function. In the modified definition of the correlation coefficient used in this work, several essential features of the correlation analysis are still maintained. First, anti-correlation manifests in a negative value of the correlation coefficient, which is utilized to suppress vessel structures selectively with anti-correlated signal behavior by setting all negative values to zero. Second, static background signal with no variation in enhancement over time is maximally suppressed. This is particularly important in fast 3D acquisitions, in which short repetition times are achieved by reducing the duration of the radiofrequency pulses, which results in increased background signal intensities at the margin of the 3D slab. These signal inhomogeneities substantially limit evaluation of the whole 3D volume by the MIP algorithm. Third, an improved signalto-noise ratio can be achieved in arterial and venous correlation maps, because all images of the time series are used for data evaluation, compared with standard image subtraction of the arterial from the non-en- hanced or arteriovenous phase, in which only two data sets are used.

Discrimination between vessels with an anti-correlating enhancement pattern increases the more time points are available. In fast $2 \mathrm{D}$ acquisitions with sub-second acquisition times, hundreds of different time points can be obtained for a particular region of interest. With 3D techniques, only a few time frames are acquired in a breath-hold. Nevertheless, the anti-correlating pattern created by the combination of four different time frames and dual-bolus injection proved to be sufficient for arterial and venous correlation maps.

The combined approach presented (multiphase angiography, dual-bolus injection, and correlation analysis) allows creation of pure arterio- and venograms with decent anatomic coverage and a spatial resolution of about $2 \mathrm{~mm}$. In the reader analysis the vessel patency could be evaluated to the level of the segmental vessels. Most segmental vessels still revealed the maximum vessel conspicuity score with definitive evaluation of patency. Only the most anterior and posterior vessel segments were not reliably seen. This is related to the limited anatomic coverage of the 3D slab. In addition, cardiac motion substantially affected assessment of the lingula and left lower lobe vessels. Breathing artifacts were usually considered mild, since all patients could hold their breath during the total scan time of 24 seconds. However, this might be biased since rather young patients were studied who were not acutely ill. In none of the patients did substantial venous overlay occur in the pulmonary arteriograms.

The most challenging part of the technique was exact initiation of the scan during maximum arterial enhancement in the pulmonary artery. Particularly when the first phase proved to be timed too early, the subsequent phases would not follow the intended enhancement pattern. Automated or semiautomated detection of contrast media arrival such as the Smartprep technique (13) or MR fluoroscopy (14) might be helpful to overcome this limitation. Alternatively, the frame rate could be increased to acquire at least one optimally enhanced arterial phase for each bolus administered. Radial projection k-space acquisition schemes are particularly suited to reconstruct multiple time frames from a limited number of views (15). However, so far these techniques require off-line post-processing and might be prone to streak artifacts.

The diagnostic workup of many pulmonary diseases could be facilitated with a non-invasive, safe technique that allows pulmonary arterio- and venograms to be acquired in a single breath-hold. Surgical planning could benefit from the selective 3D visualization of arteries and veins compressed or invaded by centrally growing tumors. The different components of arteriovenous malformations including feeding and draining vessels could be selectively visualized, and the rate of contrast fill-in and transit could be assessed. This also includes monitoring of the lesion after interventional embolization. Selective venograms are particularly useful to assess the pulmonary venous system for thrombi. In patients status post cerebrovascular accident of unknown etiology, pulmonary venous thrombi have 
been suspected as a result of local stasis after recent pneumonia. Currently, these patients face indefinite long-term anticoagulation without proof of any underlying cause of emboli. In our ongoing study, no pulmonaryvenous thrombi have been detected so far, which could be related to the small number of patients studied. In addition, only veins up to the order of segmental branches with diagnostic vessel visibility were evaluated for definitive criteria of thrombus. Small thrombi in subsegmental veins as well as in larger veins with poorer vessel visibility might be overlooked.

In summary, the combination of time-resolved multiphasic acquisition, modified infusion protocols tailored to exact enhancement kinetics of the vascular territory, and dedicated post-processing such as correlation analysis is able to visualize different pulmonary vascular segments selectively during a single breath-hold exam. The spatial resolution and anatomic coverage are already decent. If it is further improved, this technique will represent a strong competitor for conventional $\mathrm{X}$-ray digital subtraction angiography.

\section{ACKNOWLEDGMENTS}

The authors thank M. Blasche, MS, and S. Gröninger, MS, for technical support as well as Frank Londy, RT, for help with the data analysis. This work was supported by grants from the Deutsche Forschungsgemeinschaft to S.O.S. and the Tumorzentrum Heidelberg/ Mannheim (M.B.).

\section{REFERENCES}

1. Prince MR, Narasimham DL, Stanley JC, et al. Breath-hold gadolinium-enhanced MR angiography of the abdominal aorta and its major branches. Radiology 1995; 197:785-792.

2. Meaney JFM, Weg JG, Chenevert TL, et al. Diagnosis of pulmonary embolism with magnetic resonance angiography. N Engl J Med 1997;336:1422-1427.
3. Nichtweiss M, Wiegand C, Hundsdorf W. Cerebral ischemia in young adults. Typical causes and diagnostic problems. Nervenarzt 1990;61:472-481

4. Sloop RD, Lium JH. Systemic arterial embolism arising from pulmonary thrombophlebitis. Am Surg 1971;37:503-505.

5. Schoenberg SO, Knopp MV, Grau A, et al. Ultrafast MR venography in the lungs. Radiologe 1998;38:597-605.

6. Schoenberg SO, Bock M, Knopp MV, et al. Renal arteries: optimization of 3D gadolinium MR angiography with bolus-timing independent fast multiphase acquisition in a single breath hold. Radiology 1999;211:667-679.

7. Schoenberg SO, Knopp MV, Prince MR, Londy F, Knopp MA. Arterial phase three-dimensional gadolinium magnetic resonance angiography of the renal arteries: strategies for timing and contrast media injection. Invest Radiol 1998;33:506-514.

8. Bock M, Schoenberg SO, Floemer F, et al. Artery-vein separation in 3D contrast enhanced pulmonary MRA using correlation analysis (abstract). In: Proceedings of the Seventh Meeting of the International Society for Magnetic Resonance in Medicine, Berkeley, CA, 1999. p 486.

9. Bock M, Schoenberg SO, Floemer F, et al. Separation of arteries and veins in 3D MR angiographies using correlation analysis. Magn Reson Med (in press).

10. Korosec FR, Frayne R, Grist TM, Mistretta CA. Time-resolved contrast-enhanced 3D MR angiography. Magn Reson Med 1996;36: 345-351.

11. Bandettini PA, Jesmanowicz A, Wong EC, Hyde JS. Processing strategies for time-course data sets in functional MRI in the human brain. Magn Reson Med 1993;30:161-173.

12. Strecker R, Lehnhardt S, Klisch J, Hennig J. Functional MRA combining 2D MRA DSA and correlation analysis (abstract). In: Proceedings of the Seventh Meeting of the International Society for Magnetic Resonance in Medicine, Berkeley, CA, 1999. p 484.

13. Prince MR, Chenevert TL, Foo TKF, et al. Contrast-enhanced abdominal MR angiography: optimization of imaging delay time by automating the detection of contrast material arrival in the aorta. Radiology 1997;203:109-114.

14. Wilman AH, Riederer SJ, King BF, et al. Fluoroscopically triggered contrast-enhanced three-dimensional MR angiography with elliptical centric view order: application to the renal arteries. Radiology 1997;205:137-146.

15. Vigen KK, Peters DC, Grist TM, Block WF, Mistretta CA. Undersampled projection imaging for time-resolved contrast-enhanced 3D MR angiography (PR-TRICKS) (abstract). In: Proceedings of the Seventh Meeting of the International Society for Magnetic Resonance in Medicine, Berkeley, CA, 1999. p 1899. 